\title{
Die Nutzung von Hilfe und Unterstützungsangeboten von verschiedenen Gruppen von Kokaingebrauchern in Zürich
}

\section{F. Güttinger \\ J. Rehm}

\author{
Utilization of Health and Social Support Services by Different Groups of Cocaine \\ Users in Zurich, Switzerland
}

\section{Zusammenfassung}

Zielsetzung: In Zürich wie auch in vielen anderen Großstädten Europas bestehen trotz der zunehmenden Kokainproblematik kaum spezifische Hilfs- und Unterstützungsangebote für Kokainkonsumierende. Die Befragung von Konsumierenden zu ihren Behandlungserfahrungen und -bedürfnissen sollte zeigen, wie bestehende Angebote verbessert und wo neue zielgruppenspezifische Hilfe und Unterstützung notwendig sind. Methode: Es wurden im Jahr 2002 in Zürich drei Zielgruppen von Kokainkonsumierenden mittels eines umfassenden Fragebogens in einer Face-to-face-Befragung interviewt: Personen in drogenspezifischer Behandlung, vorwiegend Methadon $(n=70)$, nicht behandelte, desintegrierte Personen aus der Drogenszene $(n=61)$ und Personen aus dem Umfeld der Partyszene $(n=70)$. Ergebnisse: Kokainkonsumierende aus dem Umfeld der Partyszene haben wenig Erfahrung mit Behandlungen. Sie fühlen sich in ihrem Alltag zwar kaum durch Drogenprobleme beeinträchtigt, scheinen aber trotzdem unter körperlichen Folgen zu leiden. Rund ein Drittel der Personen in drogenspezifischer Behandlung hat sich bereits wegen seiner Kokainprobleme in der einen oder anderen Form behandeln lassen; in etwas geringerem Maße trifft das auf Personen aus der Drogenszene zu. Für knapp die Hälfte der Personen beider Gruppen ist eine Behandlung der Drogenprobleme sehr wichtig. Da für die Behandlung der Opioidabhängigkeit entsprechende Angebote zur Verfügung stehen, dürfte sich dieser Wunsch bei den häufig mehrfachabhängigen Befragten haupt-

\section{Abstract}

Objective: In Zurich as in other metropolitan cities in Europe, there are not sufficient health and social support services to cope with the increasing needs of cocaine users. A survey of cocaine users on their treatment and support needs was conducted to help improve the current and indicate the need for new services. Methods: In the year 2002, three groups of cocaine consumers were interviewed on their treatment and support needs face to face based on a structured questionnaire: cocaine users in drug-treatment, mainly methadone maintenance treatment $(n=70)$, untreated, desintegrated users from the drug scene $(n=61)$ and users from the party scene $(n=70)$. Results: People consuming cocaine from the party scene have few experience with treatment. Although they claim to be rarely impaired in daily life by their drug use, they seem to experience problems with the somatic consequences. About one third of the people in opioid and other drug treatment have been treated for cocaine problems, as well as a somewhat smaller portion of people from the drug scene. About one half of the members of both groups state that treatment for drugs problems is very important for them. Given the availability of sufficient and diversified treatment for opioid treatment, this demand refers mainly to treatment of the cocaine problem. Conclusion: As cocaine users in the party scene do not seek help from the drug-specific treatment and social support system, family doctors may be the right venue to deliver services. Thus, family doctors should be sensi-

Institutsangaben

Institut für Suchtforschung, Zürich, Schweiz

Danksagung

Wir danken dem Bundesamt für Bildung und Wissenschaft (BBW Nr. 00.0554) und dem Bundesamt für Gesundheit (Vertrag Nr. 02.000990/2.24.02.- 153) für die finanzielle Unterstützung dieses

Forschungsprojekts, das im Rahmen des EU-Forschungsprogramms Life/Generic durchgeführt wurde. Ebenfalls danken möchten wir den an dieser Studie beteiligten europäischen Projektpartnern, insbesondere dem Zentrum für Interdisziplinäre Suchtforschung der Universität Hamburg.

Korrespondenzadresse

Franziska Güttinger · Institut für Suchtforschung (ISF) · Postfach · 8031 Zürich, Schweiz ·

E-mail: franziska.guettinger@isf.unizh.ch

Bibliografie

Suchttherapie 2004; 5: 174-179 @ Georg Thieme Verlag KG Stuttgart · New York

DOI 10.1055/s-2004-813622

ISSN 1439-9903 
sächlich auf das Kokainproblem beziehen. Schlussfolgerungen: Personen, die Kokain im Partyumfeld konsumieren, suchen kaum drogenspezifische Hilfsangebote auf. Deshalb könnten Hausärzte einen möglichen Zugang zum Hilfesystem darstellen. Da die heroingestützte Behandlung nachweislich den Kokainbeikonsum reduziert, sollte Kokainkonsumierenden in Behandlung und Personen aus der Drogenszene mit gleichzeitiger Opioidabhängigkeit der Zugang zu dieser Behandlungsform erleichtert werden.

Schliuisselwörter

Kokain · Behandlung · Unterstützung tised to problems in connection to cocaine consumption. As there is evidence that heroin-assisted treatment did reduce cocaine consumptions in opioid addicts, this kind of treatment should be more accessible to cocaine addicts among multi-drug addicts.

Key words

Cocaine $\cdot$ treatment $\cdot$ support

\section{Einleitung}

Obwohl der Kokainkonsum in diversen europäischen Ländern in den letzten Jahren ein ernst zu nehmendes Problem geworden ist, sind die Hilfsangebote in den meisten Ländern Europas nach wie vor auf die Bedürfnisse von Opiatkonsumenten (insbesondere Heroinkonsumenten) ausgerichtet, da diese bisher den größten Teil der sich in Behandlung begebenden Personen ausmachten [1]. 1999 wurde im jährlichen „Report of the European Monitoring Center for Drugs and Drugs Addiction“ [2] der dringende Wunsch geäußert, Konzepte für die Behandlung von Personen mit intensivem und problematischem Kokainkonsum zu erarbeiten, denn die Nachfrage an Behandlungsmöglichkeiten für Kokainkonsumenten liegt in einigen europäischen Ländern nach den Opiaten bereits an zweiter Stelle.

Aus der Diskussion um die Behandlung von Kokainabhängigkeit wird deutlich, dass diese eine schwierig zu therapierende Suchterkrankung darstellt [3]. Aus den USA liegen diverse Studien zur pharmakologisch sowie zur psychotherapeutisch gestützten Behandlung von Kokainabhängigkeit vor. Wie die Übersichtsarbeit von Silva de Lima et al. [4] aufzeigt, liegen bis jetzt nicht genügend Daten vor, die die Wirksamkeit von Medikamenten zur Kokainbehandlung unterstützen. Im Bereich der psychotherapeutischen Behandlungen haben sich unter anderen kognitiv-behaviorale Therapien und Kontingenzmanagement als wirksam bei verschiedenen Gruppen von Kokainabhängigen erwiesen [5]. Preuss et al. [6] kommen jedoch beim Vergleich diverser Studien zur Kokainbehandlung zum Schluss, dass sich bisher keine der verschiedenen Therapieformen als klar überlegen herausgestellt hat. Auf internationaler Ebene hat sich vor allem das US National Institute on Drug Addiction mit der Entwicklung von Behandlungsprogrammen für diesen Typ von Drogenmissbrauch hervorgetan. Der Schwerpunkt ihrer Forschungsbemühungen liegt auf der Entwicklung einer Medikation sowie der Erforschung von Verhaltenstherapien [7].

In der Schweiz werden zwar im Rahmen der Schweizerischen Gesundheitsbefragung empirische Daten zur Prävalenz des Kokaingebrauchs erhoben [8, 9], jedoch klammern diese Daten das Dunkelfeld aus [10] und es fehlen insbesondere Daten zum problematischen Kokaingebrauch. Bisher werden in der Schweiz noch keine spezifischen Behandlungen für Kokainabhängige als fester Bestandteil des Therapiespektrums angeboten. Mangels Alternativen ist ein Teil der Kokainabhängigen deshalb in unterschiedlichen Behandlungsprogrammen substanzbedingter Er- krankungen zu finden. Ein Großteil der bestehenden Angebote für Drogenabhängige wurde bei seiner Planung auf Heroinkonsumierende ausgerichtet $[11,12]$. Viele dieser Personen weisen jedoch politoxikomane Konsummuster auf. Dabei sind der Kokainkonsum sowie der gleichzeitige Gebrauch von Opiaten (Heroin, Methadon) mit Kokain gemäß Beobachtungen von Fachleuten inzwischen weit verbreitet $[3,13]$. Dies bedeutet aber auch, dass sich die Hilfs- und Unterstützungsangebote auf neue Zielgruppen einstellen müssen.

Wie sich zeigt, wird Kokain von unterschiedlichen Gruppen in unterschiedlichen Kontexten konsumiert [14]. Nicht nur in der so genannten Drogenszene, sondern auch in der (Techno-)Partyszene ist Kokain stark verbreitet. Ein Vergleich einer Stichprobe von Partygängern mit Gleichaltrigen der Durchschnittsbevölkerung ergab einen um ein Vielfaches höheren Anteil an Personen mit Kokainkonsumerfahrung [15]. Die berechtigte Angst besteht, dass sich bei einigen dieser Personen der sporadische Konsum zu einer Abhängigkeit entwickelt [13].

Im Rahmen eines multizentrischen Projekts haben sich neun europäische Großstädte zusammengeschlossen, die durch ihre Kooperation dem zunehmenden Kokainproblem begegnen und die Angemessenheit des Behandlungs- und Hilfesystems beurteilen wollen. Dieses Forschungsprojekt hat es sich zur Aufgabe gemacht, einerseits die Gruppe von Kokain- und Crackkonsumierenden und ihrer spezifischen Probleme zu beschreiben, andererseits bestehende Unterstützungsangebote zu analysieren [16].

Im vorliegenden Artikel werden die Ergebnisse einer quantitativen Konsumentenbefragung in der Stadt Zürich in Bezug auf die Nutzung von bestehenden Hilfsangeboten wiedergegeben. Ziel ist es, die speziellen Behandlungsbedürfnisse für verschiedene Subgruppen von Kokainkonsumierenden zu eruieren sowie Empfehlungen für die Verbesserung von Hilfsangeboten zu formulieren.

\section{Methodik}

\section{Stichprobe}

In die Befragung einbezogen wurden Personen, die gemäß eigenen Angaben im letzten Monat vor dem Befragungszeitpunkt mindestens einmal Kokainpulver, Crack oder Freebase konsumiert hatten. Drei verschiedenen Zielgruppen von Kokain- 
oder CrackkonsumentInnen wurden für die Studie interviewt. Sie sind wie folgt definiert:

- Gruppe 1 („Behandlungsgruppe“): Kokain- oder Crackkonsumierende, die sich in einer drogenspezifischen Behandlung befinden, hauptsächlich ambulante Substitutionstherapie (v. a. Substitutionsbehandlungen wie Methadonbehandlung, Heroinverschreibung); $\mathrm{n}=70$;

- Gruppe 2 („Drogenszenegruppe“): Kokain- oder Crackkonsumierende, die der Drogenszene angehören, d.h. hauptsächlich desintegrierte Personen, die sich in keiner drogenspezifischen Behandlung befinden; $\mathrm{n}=61$;

- Gruppe 3 („Partygruppe“): Kokain- oder Crackkonsumierende aus dem Umfeld der Partyszene, die nicht der Drogenszene angehören und sich auch in keiner drogenspezifischen Behandlung befinden; $\mathrm{n}=70$.

Gruppe 1 („Behandlungsgruppe“) unterscheidet sich von Gruppe 2 („Drogenszenegruppe“) und Gruppe 3 („Partygruppe“) durch ihre Einbindung in eine Behandlung, während Gruppe 2 und Gruppe 3 in unterschiedlichem Ausmaß sozial integriert sind. Die Drogenszenegruppe wird als sozial desintegriert und die Partygruppe als sozial integriert definiert.

Die Probanden aus Gruppe 1 befinden sich mehrheitlich in Methadonbehandlung. Da es sich als schwierig erwies, in Zürich genügend Kokainkonsumierende zu finden, die sich nicht in einer drogenspezifischen Behandlung befinden, wurde die Rekrutierung für Gruppe 2 bei 61 Personen abgebrochen.

Das ganze Sample basiert auf der Selbstselektion von Teilnehmenden.

\section{Durchführung der Befragung}

Die drei verschiedenen Zielgruppen von Kokain- und Crackkonsumierenden wurden an unterschiedlichen Orten, die einen guten Zugang zu den einzelnen Gruppen erlaubten, kontaktiert. Die Probanden aus Gruppe 1 (Behandlung) und Gruppe 2 (Drogenszene) wurden bei diversen niederschwelligen Angeboten für Drogenabhängige (Kontakt- und Anlaufstellen, Arbeitsangebote für Randständige, ambulante Arztpraxis zur medizinischen Grundversorgung für Obdachlose und Randständige, Spritzentauschstellen) rekrutiert. Die Interviews mit Personen aus der Partyszene kamen in einschlägigen Lokalen, auf Partys und Treffpunkten zustande, vorwiegend aber über persönliche Kontakte.

Die Befragung erfolgte face-to-face mittels eines standardisierten Fragebogens, wobei ein kurzer Fragenkomplex in Form einer Selbsteinschätzung von den Interviewten selbst ausgefüllt wurde. Der Fragebogen umfasste diverse standardisierte Instrumente. Die vorliegende Auswertung basiert auf Fragen aus dem „Maudsley Addiction Profile MAP“ [17], dem EuropASI [18] sowie weiteren Fragen zur sozialen Situation und zur gegenwärtigen Benutzung von Behandlungen und Beratungen.

Die Datenerhebung dauerte von Juni bis November 2002 und erfolgte ausschließlich in der Stadt Zürich.

\section{Datenauswertung}

Die Auswertung der Daten erfolgte quantitativ mittels SPSS 11 für Windows. Beim Vergleich von Merkmalen der verschiedenen Gruppen kam der $\chi^{2}$-Test zur Anwendung. Einfaktorielle ANOVA und der Scheffé-Test mit Bonferroni-Adjustierung wurden für Mittelwertsvergleiche herangezogen.

\section{Ergebnisse}

\section{Soziodemografische Daten}

Die wichtigsten soziodemografischen Daten sind aus Tab. 1 ersichtlich. Der Frauenanteil von einem Fünftel bis einem Viertel in der Gruppe der Behandelten und der Nichtbehandelten aus der Drogenszene entspricht ihrem Anteil bei anderen Datenerhebungen unter Drogenabhängigen. Einen beträchtlich höheren Prozentanteil machen Frauen mit 37\% in der Partygruppe aus. Das Durchschnittsalter in allen drei Gruppen beträgt zwischen 33 und 34 Jahren.

Tab. 1 Soziodemografische Daten und soziale Integration

\begin{tabular}{|c|c|c|c|c|}
\hline & $\begin{array}{l}\text { Gruppe 1: } \\
\text { Behandlung } \\
n=70\end{array}$ & $\begin{array}{l}\text { Gruppe } 2 \\
\text { Drogenszene } \\
n=61\end{array}$ & $\begin{array}{l}\text { Gruppe } 3 \\
\text { Partyszene } \\
n=70\end{array}$ & $\begin{array}{l}\text { total } \\
n=201\end{array}$ \\
\hline $\begin{array}{l}\text { Geschlecht } \\
\text { - männlich } \\
\text { - weiblich }\end{array}$ & $\begin{array}{l}79 \%(55) \\
21 \%(15)\end{array}$ & $\begin{array}{l}75 \%(46) \\
25 \%(15)\end{array}$ & $\begin{array}{l}63 \%(45) \\
37 \%(26)\end{array}$ & $\begin{array}{l}72 \%(146) \\
28 \%(56)\end{array}$ \\
\hline $\begin{array}{l}\text { Alter } \\
\text { Standard- } \\
\text { abweichung } \\
\text { - Männer } \\
\text { - Frauen }\end{array}$ & $\begin{array}{l}34 \text { Jahre } \\
7 \text { Jahre } \\
35 \text { Jahre } \\
33 \text { Jahre }\end{array}$ & $\begin{array}{l}8 \text { Jahre } \\
34 \text { Jahre } \\
30 \text { Jahre }\end{array}$ & $\begin{array}{l}7 \text { Jahre } \\
34 \text { Jahre } \\
30 \text { Jahre }\end{array}$ & $\begin{array}{l}7 \text { Jahre } \\
34 \text { Jahre } \\
33 \text { Jahre }\end{array}$ \\
\hline $\begin{array}{l}\text { abgeschl. Be- } \\
\text { rufsausbildung }\end{array}$ & $63 \%(44)$ & $59 \%(36)$ & $78 \%(54)$ & $67 \%(134)$ \\
\hline
\end{tabular}

\section{Soziale Situation}

Auffallend viele Personen in Behandlung (16\%) und aus der Drogenszene (23\%) sind obdachlos. Der Unterschied zur Gruppe der

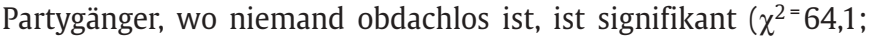
$\mathrm{df}=6 ; \mathrm{p}<0,000)$. Auch hinsichtlich der Arbeitssituation zeigen sich signifikante Unterschiede zwischen den einzelnen Gruppen von Kokainkonsumenten. Während bei Gruppe 1 und 2 rund 70\% der Personen arbeitslos oder arbeitsunfähig sind, sind dies bei Gruppe 3 zwar deutlich weniger, jedoch insgesamt immer noch $20 \%\left(\chi^{2}=46,7 ; \mathrm{df}=6 ; \mathrm{p}<0,000\right)$. Starke Unterschiede zwischen Gruppe 1 und 2 einerseits und Gruppe 3 andererseits zeigen sich ebenfalls bezüglich sozialer Kontakte. Etwas mehr Personen aus dem Umfeld der Partyszene leben zwar alleine, die häufigeren Kontakte zu Partnern und nahe stehenden Freunden sind jedoch ein weiteres Zeichen ihrer besseren sozialen Integration im Vergleich zu Kokainkonsumierenden aus den beiden anderen Gruppen.

\section{Ausmaß der Drogenprobleme}

Bezüglich des Ausmaßes der Drogenprobleme sind ebenfalls signifikante Unterschiede zwischen Behandelten sowie Nichtbehan- 
delten aus der Drogenszene einerseits und den Partygängern feststellbar. Personen aus Gruppe 1 und 2 haben an markant mehr Tagen Drogenprobleme und sind erheblicher durch diese belastet als die Partygänger $(F=18,5 ; d f=2 ; p<0,000$, Scheffé-Test signifikant). Außerdem sind 45 bzw. $46 \%$ von ihnen die (zusätzliche) Behandlung dieser Drogenprobleme sehr wichtig (Tab. 3).

\section{Behandlungserfahrungen}

Wohnsituation ${ }^{1}$

- mit anderen Personen

- alleine

- in Institution/sonstige

- ohne feste Unterkunft

Arbeitssituation ${ }^{1}$

- Voll-/Teilzeitarbeit

- temporär, Ausbildung

- arbeitslos

- krank, arbeitsunfähig,

Rentner/In

Häufigkeit der Kontakte mit

- Partnerln (falls vorhan-

den) $)^{2}$

- Verwandten

- nahe stehenden Freunden ${ }^{1}$

${ }^{1} p<0,000 ;{ }^{2} p<0,05$

Tab. 3 Ausmaß der Drogenprobleme

\begin{tabular}{|c|c|c|c|c|}
\hline & $\begin{array}{l}\text { Gruppe } 1 \\
\text { Behand- } \\
\text { lung }\end{array}$ & $\begin{array}{l}\text { Gruppe } 2 \\
\text { Drogen- } \\
\text { szene }\end{array}$ & $\begin{array}{l}\text { Gruppe } 3 \\
\text { (Party- } \\
\text { szene) }\end{array}$ & total \\
\hline Tage mit Drogenproblemen ${ }^{1}$ & 22 Tage & 24 Tage & 8 Tage & 21 Tage \\
\hline Standardabweichung & $\begin{array}{l}11 \text { Tage } \\
\mathrm{n}=61\end{array}$ & $\begin{array}{l}10 \text { Tage } \\
\mathrm{n}=55\end{array}$ & $\begin{array}{l}10 \text { Tage } \\
n=20\end{array}$ & $\begin{array}{l}12 \text { Tage } \\
\mathrm{n}=136\end{array}$ \\
\hline $\begin{array}{l}\text { erhebliche Belastung durch } \\
\text { Drogenprobleme }^{1}\end{array}$ & $\begin{array}{l}62 \%(41) \\
n=66\end{array}$ & $\begin{array}{l}53 \%(31) \\
\mathrm{n}=58\end{array}$ & $\begin{array}{l}8 \%(5) \\
n=64\end{array}$ & $\begin{array}{l}41 \%(77) \\
n=188\end{array}$ \\
\hline $\begin{array}{l}\text { Behandlung der Drogen- } \\
\text { probleme sehr wichtig }{ }^{1}\end{array}$ & $\begin{array}{l}46 \%(30) \\
n=66\end{array}$ & $\begin{array}{l}45 \%(26) \\
n=58\end{array}$ & $\begin{array}{l}3 \%(2) \\
n=64)\end{array}$ & $\begin{array}{l}31 \%(58) \\
n=188\end{array}$ \\
\hline
\end{tabular}

${ }^{1} \mathrm{p}<0,000$

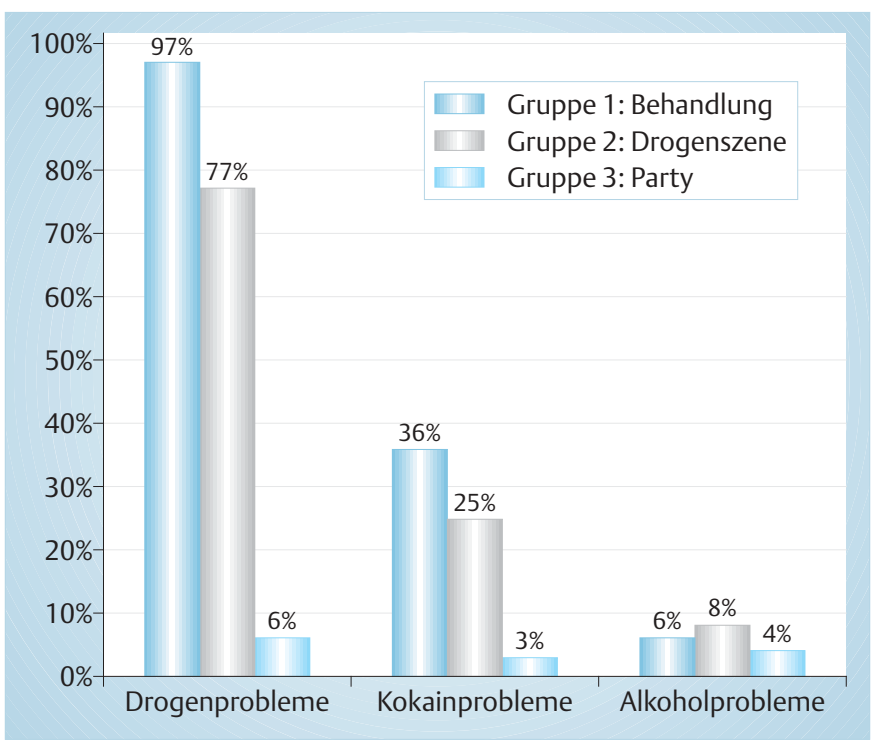

Abb. 1 Erfahrene Behandlungen

$\begin{array}{lccc}23 \%(16) & 35 \%(21) & 54 \%(38) & 38 \%(75) \\ 36 \%(25) & 37 \%(22) & 44 \%(31) & 39 \%(78) \\ 26 \%(18) & 5 \%(3) & 1 \%(1) & 11 \%(22) \\ 16 \%(11) & 23 \%(14) & 0 \%(0) & 13 \%(25) \\ & & & \\ 12 \%(8) & 20 \%(12) & 67 \%(44) & 33 \%(64) \\ 15 \%(10) & 10 \%(6) & 14 \%(9) & 13 \%(25) \\ 37 \%(25) & 47 \%(28) & 18 \%(12) & 34 \%(65) \\ & & & \\ 37 \%(25) & 23 \%(14) & 2 \%(1) & 21 \%(40) \\ & & & \\ 7 \text { Tage } & \text { 7 Tage } & \text { 12 Tage } & \text { 9 Tage } \\ \text { 5 Tage } & \text { 7 Tage } & \text { 4 Tage } & \text { 5 Tage } \\ \text { 7 Tage } & \text { 9 Tage } & \text { 24 Tage } & \text { 14 Tage } \\ & & & \end{array}$

Über ein Drittel der Behandelten und ein Viertel der Personen aus der Drogenszene waren schon mindestens einmal hauptsächlich wegen Problemen mit Kokain oder Crack in Behandlung (Abb.1). Am häufigsten wurde in Form eines stationären Entzugs und/oder einer Therapie versucht, vom Kokain loszukommen. Daneben nennen rund 10\% von Gruppe 1 und von Gruppe 2 die Behandlung mit Antidepressiva und fast ebenso viele haben sich Verhaltens- bzw. Psychotherapie unterzogen (nicht in Tabelle). Von den Kokainkonsumierenden aus der Partyszene war hingegen bisher kaum jemand in Behandlung oder hat einen Entzug bzw. eine stationäre Therapie gemacht.

Fast ein Drittel von Gruppe 1 hat bereits mehr als fünf Behandlungen wegen Drogenproblemen und jeder Zehnte gibt an, schon mehr als fünf Behandlungen wegen Kokain und/oder Crack gemacht zu haben (Abb. 2). Personen aus Gruppe 1 haben bereits mehr Behandlungserfahrung in Bezug auf Drogen als Personen aus der Drogenszene.

\section{Nutzung von Beratungs- und Behandlungsangeboten}

Bei der Frage, was für Beratungs- oder Behandlungsangebote in den letzten 30 Tagen in Anspruch genommen wurden, ergaben sich fast durchwegs signifikante Unterschiede zwischen den Gruppen 1 bzw. 2 und Gruppe 3 (Tab. 4). Von den Partygängern stand kaum jemand wegen psychischer, beruflicher, finanzieller, rechtlicher, sozialer Probleme sowie Wohnproblemen in Behandlung oder Beratung. Eine Ausnahme bilden körperliche Probleme. Deswegen stand rund die Hälfte der Behandelten sowie immerhin ein Viertel der Befragten aus der Partyszene und ebenso viele aus der Drogenszene in Behandlung. Zusätzlich zur Nutzung der Angebote interessierte, wie wichtig den Kokainkonsumenten aktuell eine Beratung oder Behandlung wegen diverser (körperlicher, emotional-psychischer oder Alkohol- oder Drogen-)Probleme ist. 65\% der Partygänger gaben an, dass ihnen zurzeit eine Beratung oder Behandlung ihrer körperlichen Probleme erheblich bis extrem wichtig ist. Ein etwa gleich hoher Prozentsatz der Partygänger stufte die Behandlung

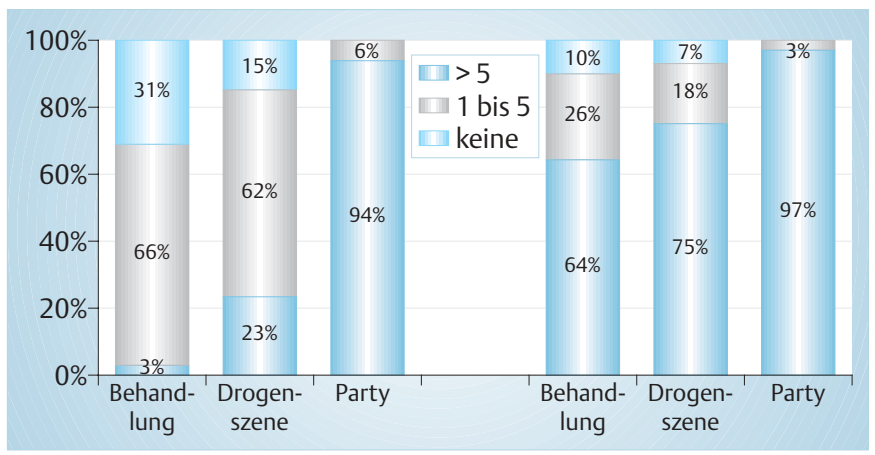

Abb. 2 Anzahl Behandlungen wegen Drogenproblemen und Kokain-/ Crackproblemen 
Tab. 4 Nutzung von Beratungs- und Behandlungsangeboten in den letzten 30 Tagen

\begin{tabular}{|c|c|c|c|c|}
\hline & $\begin{array}{l}\text { Gruppe } 1 \\
\text { Behand- } \\
\text { lung }\end{array}$ & $\begin{array}{l}\text { Gruppe } 2 \\
\text { Drogen- } \\
\text { szene }\end{array}$ & $\begin{array}{l}\text { Gruppe } 3 \\
\text { Partyszene }\end{array}$ & total \\
\hline $\begin{array}{l}\text { in Beratung/Behandlung } \\
\text { wegen körperlicher Pro- } \\
\text { bleme }\end{array}$ & $\begin{array}{l}51 \%(36) \\
n=70\end{array}$ & $\begin{array}{l}25 \%(15) \\
n=61\end{array}$ & $\begin{array}{l}23 \%(16) \\
n=70\end{array}$ & $\begin{array}{l}33 \%(67) \\
n=201\end{array}$ \\
\hline $\begin{array}{l}\text { in Beratung/Behandlung } \\
\text { wegen emotionaler, } \\
\text { psychischer Probleme }\end{array}$ & $\begin{array}{l}20 \%(14) \\
n=70\end{array}$ & $\begin{array}{l}23 \%(14) \\
n=60\end{array}$ & $\begin{array}{l}7 \%(5) \\
n=70\end{array}$ & $\begin{array}{l}17 \%(33) \\
n=200\end{array}$ \\
\hline $\begin{array}{l}\text { in Beratung/Behandlung } \\
\text { wegen Alkohol-/Dro- } \\
\text { genproblemen }\end{array}$ & $\begin{array}{l}99 \%(69) \\
n=70\end{array}$ & $\begin{array}{l}26 \%(16) \\
n=61\end{array}$ & $\begin{array}{l}1 \%(1) \\
\mathrm{n}=70\end{array}$ & $\begin{array}{l}43 \%(86) \\
n=201\end{array}$ \\
\hline $\begin{array}{l}\text { Beratung/Behandlung } \\
\text { wegen körperlicher } \\
\text { Probleme sehr wichtig }\end{array}$ & $\begin{array}{l}49 \%(34) \\
n=70\end{array}$ & $\begin{array}{l}36 \%(22) \\
\mathrm{n}=61\end{array}$ & $\begin{array}{l}65 \%(41) \\
n=63\end{array}$ & $\begin{array}{l}50 \%(97) \\
n=194\end{array}$ \\
\hline $\begin{array}{l}\text { Beratung/Behandlung } \\
\text { wegen emotionaler, } \\
\text { psychischer Probleme } \\
\text { sehr wichtig }\end{array}$ & $\begin{array}{l}49 \%(34) \\
n=70\end{array}$ & $\begin{array}{l}46 \%(28) \\
n=61\end{array}$ & $\begin{array}{l}65 \%(41) \\
n=63\end{array}$ & $\begin{array}{l}53 \%(103) \\
n=194\end{array}$ \\
\hline $\begin{array}{l}\text { Beratung/Behandlung } \\
\text { wegen Alkohol-/Dro- } \\
\text { genproblemen sehr } \\
\text { wichtig }\end{array}$ & $\begin{array}{l}99 \%(69) \\
n=70\end{array}$ & $\begin{array}{l}26 \%(16) \\
n=61\end{array}$ & $\begin{array}{l}1 \%(1) \\
\mathrm{n}=70\end{array}$ & $\begin{array}{l}43 \%(86) \\
n=201\end{array}$ \\
\hline
\end{tabular}

${ }^{1} \mathrm{p}<0,000$

von psychischen Problemen sowie von Alkohol- oder Drogenproblemen als sehr wichtig ein.

\section{Zusammenfassung und Diskussion}

Spezifische, auf Kokainkonsumierende zugeschnittene Hilfsangebote gibt es in der Schweiz nur vereinzelt. In den Heroinabgabestellen von Bern und Basel startete im Juni 2004 ein Pilotversuch mit der Abgabe von Ritalin zur Behandlung von Personen, die Kokain und Heroin konsumieren. Außerdem verfügen in der Stadt Zürich seit kurzem alle Kontakt- und Anlaufstellen über Raucherräume auch für Kokainkonsumierende. Ziel der vorliegenden Analyse war es, die Inanspruchnahme von Hilfe, die Behandlungserfahrungen und die Unterstützungsbedarfe unterschiedlicher Zielgruppen von Kokainkonsumierenden zu erfassen, d.h. von Personen in Behandlung, von nicht behandelten Personen aus der Drogenszene und von Personen aus dem Umfeld der Partyszene.

Die soziodemografischen Daten zeigen, dass bei den Partygängern ein eher älteres Segment von Kokainkonsumierenden erreicht wurde. Altersmäßig entsprechen sie zwar den behandelten Personen und denjenigen aus der Drogenszene, jedoch wurde zu Beginn des Projekts ein eher jüngeres Zielpublikum anvisiert. Da sich die Rekrutierung auf Partys als eher schwierig erwies, kam ein Großteil der Interviews über persönliche Kontakte zustande, wodurch unbeabsichtigt eine eher ältere Gruppe von Partygängern befragt wurde. Folglich treffen die gemachten Beobachtungen nur beschränkt auf jüngere Personen aus der Partyszene zu. Neben der hohen Obdachlosigkeit und der schlechten Arbeitsintegration der Personen aus Gruppe 1 und 2 fällt auch die mit 18\% erstaunlich hohe Arbeitslosigkeit unter den Partygängern auf. Zum Befra- gungszeitpunkt lag die durchschnittliche Arbeitslosigkeit im Kanton Zürich bei 3,5\%. Die Überprüfung des Alters der arbeitslosen Partygänger ergab, dass Arbeitslosigkeit nicht wie erwartet ausschließlich bei den jüngeren Personen dieser Gruppe anzutreffen ist, sondern sich über alle Alterssegmente verteilt.

Bezüglich der Behandlungserfahrungen und -bedürfnisse hat sich für die unterschiedlichen Gruppen von Kokainkonsumierenden Folgendes herauskristallisiert:

Personen aus dem Umfeld der Partyszene fühlen sich wenig durch Drogenprobleme belastet. Partygänger verfügen zudem kaum über Behandlungserfahrungen bezüglich Kokain, anderer Drogen oder Alkohol. Aufgrund der in diesem Projekt ebenfalls erhobenen Daten zum Ausmaß des Kokainkonsums [19] ist davon auszugehen, dass ihr Konsum eher hedonistisch ausgerichtet ist und ihren Alltag kaum prägt, wie dies auch Estermann allgemein für gut integrierte Konsumenten illegaler Drogen festgestellt hat [20]. Jedoch erachten rund zwei Drittel der Personen aus dem Umfeld der Partyszene aktuell eine Behandlung wegen körperlicher Probleme als sehr wichtig. Ein Viertel gibt an, im Monat vor der Befragung wegen somatischer Probleme in ärztlicher Behandlung gewesen zu sein. Nicht gefragt wurde nach den Gründen, weshalb eine Behandlung als so wichtig erachtet wird. Während desintegrierte Kokainkonsumierende häufig Drogenhilfsangebote nutzen, gibt es für integrierte Kokainkonsumierende keine adäquaten Hilfsangebote und diese Klientel will meist nicht mit Opiatmissbrauchern in Verbindung gebracht werden [21]. Treten psychische oder physische Probleme auf, gehen sie allenfalls zum Hausarzt [20]. Dies bedeutet, dass Hausärzte dahingehend ausgebildet werden müssen, dass sie problematischen Drogengebrauch erkennen und mit der betroffenen Klientel entsprechend umgehen können [22].

Der Großteil der Abhängigen aus Gruppe 1 war bereits mehrfach in Behandlung, über ein Drittel sogar ein- oder mehrmals vorwiegend wegen Kokainproblemen, wobei davon ausgegangen werden kann, dass diese Behandlungen überwiegend in nicht speziell auf Kokain ausgerichteten Settings stattfanden. Personen aus der Behandlungsgruppe fühlen sich erheblich durch Drogenprobleme belastet und eine zusätzliche Behandlung dieser Drogenprobleme ist gut der Hälfte von ihnen sehr wichtig. Da sich ihre aktuelle Behandlung hauptsächlich auf den Opiatkonsum ausrichtet, dürfte die große Wichtigkeit einer zusätzlichen Behandlung mit dem Kokainproblem zusammenhängen. Die nicht behandelten Personen aus dem Umfeld der Drogenszene verfügen über eine vergleichsweise geringere Behandlungserfahrung, wobei $25 \%$ bereits wegen Kokain in Behandlung waren und 20\% einen stationären Entzug oder eine Therapie gemacht haben. Bei vielen Personen dieser beiden Gruppen dürfte eine Mehrfachabhängigkeit vorliegen, wobei häufig Heroin bzw. Methadon mit Kokain eingenommen wird.

Untersuchungen aus der heroingestützten Behandlung zeigen, dass sich bei in Behandlung stehenden Personen innerhalb von sechs Monaten ein Rückgang des Kokainbeikonsums abzeichnet [23]. Aufgrund der aktuell zur Verfügung stehenden Behandlungen ist deshalb ein erleichterter Zugang zur heroingestützten Behandlung für Personen mit einer zusätzlichen Kokainabhängigkeit zu befürworten. Obwohl die Heroinabgabe erwiesenermaßen den Beikonsum von Kokain reduziert, kann diese jedoch die Kokain- 
sucht nicht abdecken und macht deshalb längerfristig eine spezifische Behandlung der Kokainsucht notwendig.

\section{Literatur}

${ }^{1}$ Europäische Beobachtungsstelle für Drogen und Drogensucht (E.B.D.D). Jahresbericht über den Stand der Drogenproblematik in der Europäischen Union und Norwegen. Lissabon: E.B.D.D, 2002

${ }^{2}$ European Monitoring Centre for Drugs and Drug Addiction (EMCDDA), Extended annual report on the state of the drugs problem in the European Union 1999. Luxembourg: EMCDDA, 1999

${ }^{3}$ Stohler R, Dürsteler-MacFarland KM. Störungen durch Kokain und Opiate. Therapeutische Umschau 2003; 60 (6): 329-333

${ }^{4}$ Silva de Lima M, Garcia de Oliveira Soares B, Alves Pereira Reisser A Pharmacological treatment of cocaine dependence: a systematic review. Addiction 2002; 97: 931 - 949

${ }^{5}$ Caroll KM. Implications of recent research for program quality in cocaine dependence treatment. Subst Use Misuse 2000; 35 (12 -14): $2011-2030$

${ }^{6}$ Preuss UW, Bahlmann M, Koller G et al. Die Behandlung der Kokainabhängigkeit. Intoxikation, Entzug und Rückfallprophylaxe. Fortschr Neurol Psychiat 2000; 68: 224-238

${ }^{7}$ NIDA, National Institute on Drug Abuse. http://www.drugabuse.gov/ ResearchReports/Cocaine/cocaine4.html\#treatments, 31.7.2004

${ }^{8}$ Rehm J. Konsumformen und Verbreitung illegaler Drogen in der Schweiz. In: Fahrenkrug H et al (Hrsg). Illegale Drogen in der Schweiz 1990 - 1993. Zürich: Seismo, 1995

${ }^{9}$ Schweizerische Fachstelle für Alkohol- und andere Drogenprobleme, SFA/ISPA. Zahlen und Fakten zu Alkohol und anderen Drogen 1999. Lausanne: SFA/ISPA, 1999

${ }^{10}$ Kraus L, Augustin R, Frischer M et al. Estimating prevalence of problem drug use at national level in countries of the European Union and Norway. Addiction 2003; 98: 471 - 485

${ }^{11}$ Uchtenhagen A, Gutzwiller F, Dobler-Mikola A et al. Prescription of Narcotics for Heroin Addicts. Main Results of the Swiss National Cohort Study. Basel: Karger, 1999
${ }^{12}$ Falcato L, Liechti F, Stohler R et al. Die Versorgungsstruktur. Angebot und Nachfrage im Stadt-Land-Vergleich (Resultate aus der Begleitforschung der Methadonbehandlungen im Kanton Zürich - Nr.1: Juni 1996). Zürich: Sozialpsychiatrische Forschungsgruppe der Psychiatrischen Universitätsklinik Zürich, 1998

${ }^{13}$ Vogt R. Aktuelle Situation, Entwicklungen und Herausforderungen der Schadensverminderung. abhängigkeiten 2002; 3: 5-17

${ }^{14}$ Kemmesies UE. En- oder De-Kulturation: Kokain - ein aktuelles Portrait. Wiener Zeitschrift für Suchtforschung 2000; 23 (2): 21 - 31

${ }^{15}$ Tossmann P, Boldt S, Tensil MD. The Use of Drugs within the Techno Party Scene in European Metropolitan Cities. European Addiction Research 2001; 7: 2-23

${ }^{16}$ Prinzleve M, Haasen C, Zurhold $\mathrm{H}$ et al. Cocaine use in Europe - a multi-centre study: Patterns of use in different groups. European Addiction Research (im Druck)

${ }^{17}$ Marsden J, Gossop D, Stewart D et al. The Maudsley Addiction Profile. A brief instrument for treatment outcome research. National Addiction Centre. London, 1998

${ }^{18}$ Kokkevi A, Hartgers C, Blanken P et al. European Addiction Severity Index - EuropASI. 1993

${ }^{19}$ Güttinger F, Rehm J. Konsummuster bei verschiedenen Gruppen von Kokaingebrauchern in Zürich: Implikationen für das Drogenhilfesystem. Sucht (im Druck)

${ }^{20}$ Estermann J. Sozialepidemiologie des Drogenkonsums. Zu Prävalenz und Inzidenz des Heroin- und Kokaingebrauchs und dessen polizeiliche Verfolgung. Berlin: Verlag für Wissenschaft und Bildung, 1996

${ }^{21}$ Schlender JU. Therapie von Kokainabhängigen. In: Deutsche Hauptstelle gegen die Suchtgefahren (ed). Drogenpolitik und Drogenhilfe. Freiburg: Lambertus, 1991

${ }^{22}$ Konings E, Dubois-Arber F, Narring F et al. Identifying Adolescent Drug Users: Results of a National Survey on Adolescent Health in Switzerland. Journal of Adolescent Health 1995; 16: 240-247

${ }^{23}$ Gschwend P, Eschmann S, Güttinger F et al. Konsum von Kokain vor, in und nach der Heroin gestützten Behandlung in der Schweiz. Suchttherapie $2002 ; 3: 8-12$ 